\title{
A Molecular Perspective on Water Vapour Accommodation into Ice and its Dependence on Temperature
}

\author{
Daniel Schlesinger ${ }^{1,2,}{ }^{*}$, Samuel J. Lowe ${ }^{1,2}$, Tinja Olenius ${ }^{1,2,{ }^{\dagger}}$, Xiangrui Kong ${ }^{3}$, Jan B. C. \\ Pettersson ${ }^{3}$ and Ilona Riipinen ${ }^{1,2^{*}}$ \\ ${ }^{1}$ Department of Environmental Science (ACES), Stockholm University, Svante Arrhenius väg 8, SE-106 91 \\ Stockholm, Sweden \\ ${ }^{2}$ Bolin Centre for Climate Research, Stockholm University, SE-106 91 Stockholm, Sweden \\ ${ }^{3}$ Department of Chemistry and Molecular Biology, Atmospheric Science, University of Gothenburg, Kemigården \\ 4, SE-412 96 Gothenburg, Sweden \\ ${ }^{\dagger}$ Current address: Swedish Meteorological and Hydrological Institute (SMHI), Research Department, Air Quality \\ Research Unit, SE-601 76 Norrköping, Sweden \\ * Corresponding authors: \\ Daniel.Schlesinger@aces.su.se; $\underline{\text { llona.Riipinen@aces.su.se }}$
}

1. Evaluating flux models with molecular beam data

The two flux models discussed in the main text - one accounting solely for evaporative losses from the surface layer (1P), and a second also accounting for bulk accommodation surface layer desorption (2P) - were evaluated against the molecular beam data collected by Kong et al. (2014). Both $1 \mathrm{P}$ and $2 \mathrm{P}$ contain a fitted pre-factor $A$ in the interval $[0,1000]$, while $2 \mathrm{P}$ includes also a fitted bulk accommodation rate $k_{\alpha}$ in the interval $[0,500]$ molecules/s. Examples of the detected intensity of evaporated $\mathrm{D}_{2} \mathrm{O}$ as modelled by these models for temperatures $\mathrm{T}=170 \mathrm{~K}$ and $\mathrm{T}=200 \mathrm{~K}$ are shown in Fig. S1 alongside their respective measurement time series. 

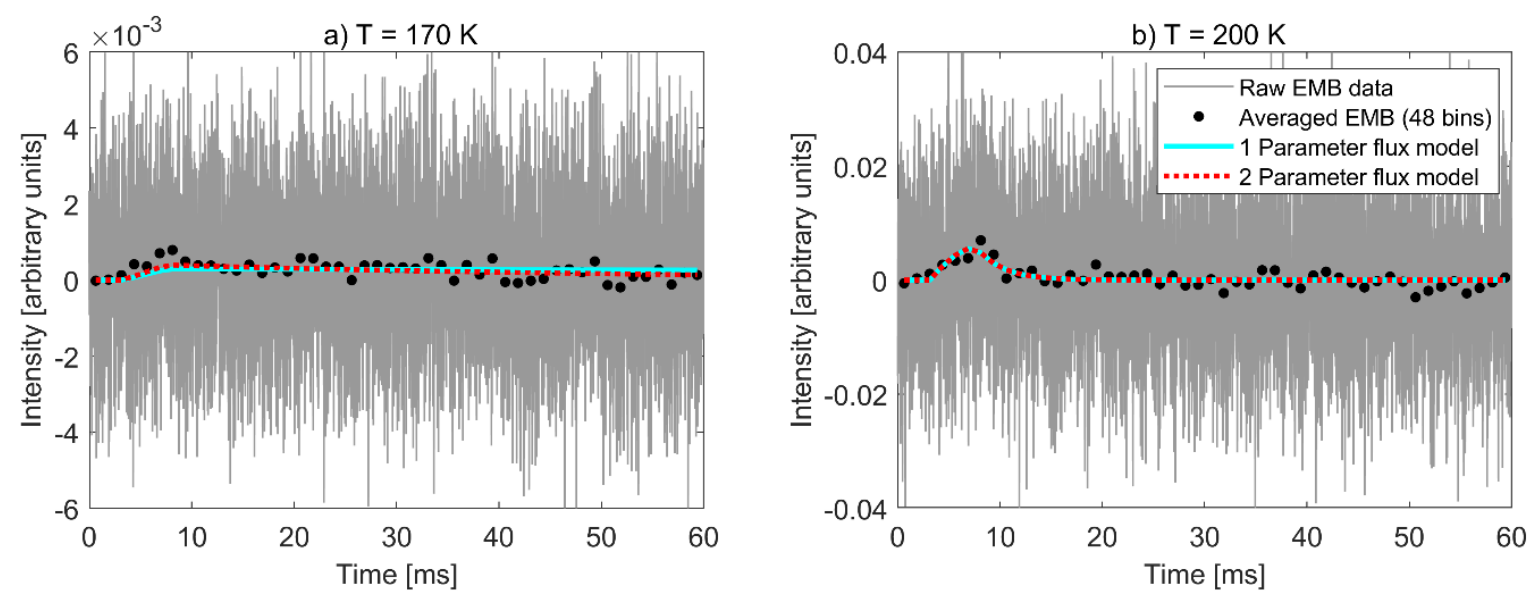

Figure S1: Evaporation only 1P (cyan) and evaporation and bulk accommodation 2P models (red) flux models for detected $\mathrm{D}_{2} \mathrm{O}$ intensity due to evaporation from the ice slab. Raw detected data is shown by the grey line and its averaged time series into 48 bins, to which the models are fit, are indicated by black dots. Panel a) is for temperature $\mathrm{T}=170 \mathrm{~K}$ and panel $\mathrm{b}$ ) for $\mathrm{T}=200 \mathrm{~K}$. 

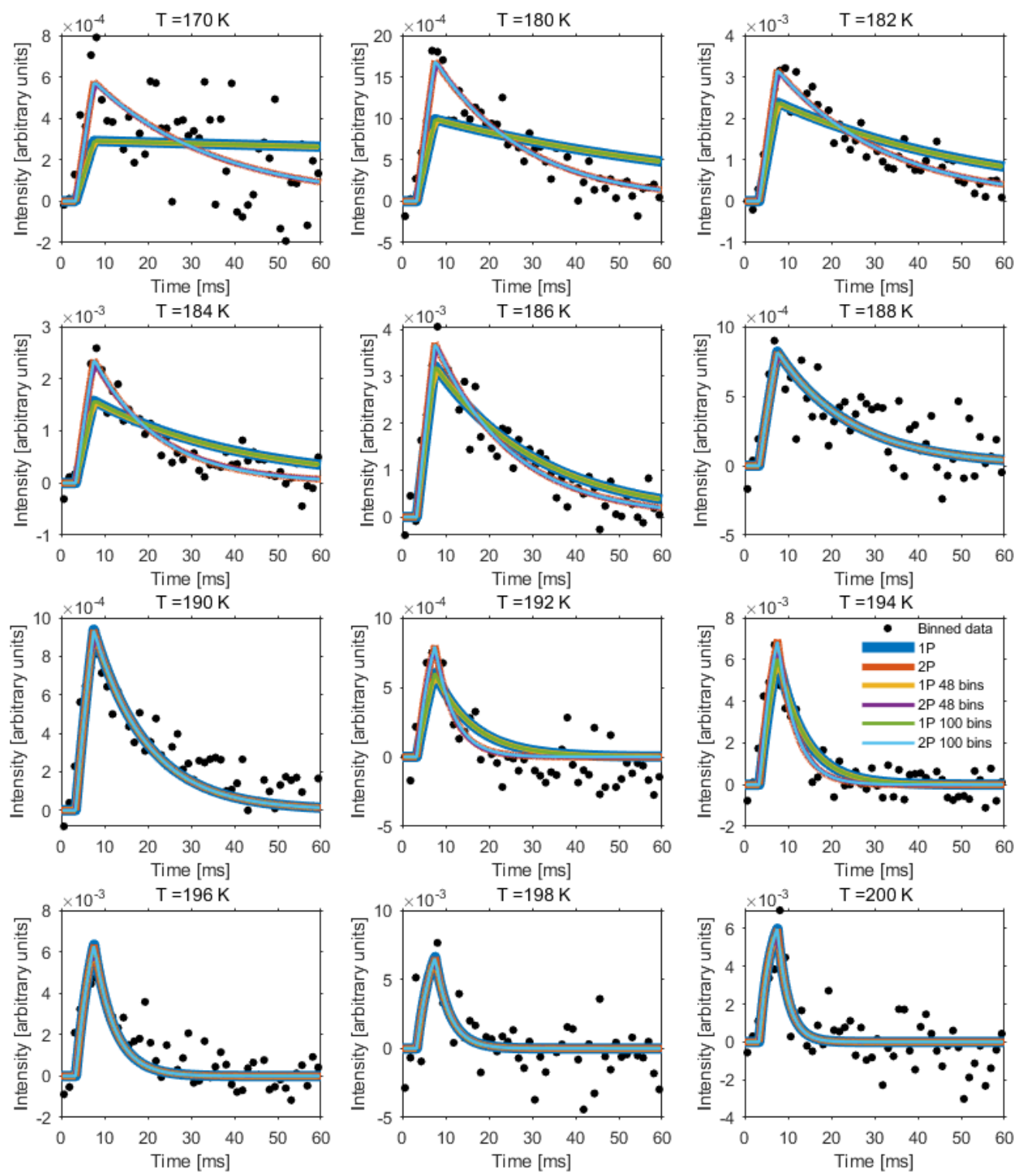

Figure S2: Curves and dots as in Figure S1 for all temperatures and including repeat fits for $1 \mathrm{P}$ and $2 \mathrm{P}$ flux models to: raw data (6000 timestamps) dark blue and orange, data binned into 48 bins yellow and purple, and 100 bins green and light blue, respectively.

Figure $\mathrm{S} 2$ shows the modelled $\mathrm{D}_{2} \mathrm{O}$ detection intensity for all temperatures found by fitting the $1 \mathrm{P}$ and $2 \mathrm{P}$ frameworks to the various time-averaged binnings given in the legend. The associated fit statistics and fitted parameters are given in Fig. S3. Differences between 1P and $2 \mathrm{P}$ schemes are more pronounced at lower temperatures $\mathrm{T}=170,180,182$ and $184 \mathrm{~K}$, and 
negligible for higher temperatures. Upon visual inspection, the resolution of the temporal averaging has no impact on the modelled intensity or fit accommodation rates (Fig. S3a). Though, as expected, this is apparent in the $\mathrm{R}^{2}$ (Fig. S3c) which increases with decreased bin number due to noise in the measurement data. This modelling was performed using a saturation vapour pressure of $\mathrm{D}_{2} \mathrm{O}$ over ice ${ }^{1}$ as it returned the best $\mathrm{R}^{2}$ statistics for the 48 -bin procedure (see Fig. S4 for comparison with other saturation vapour pressures).
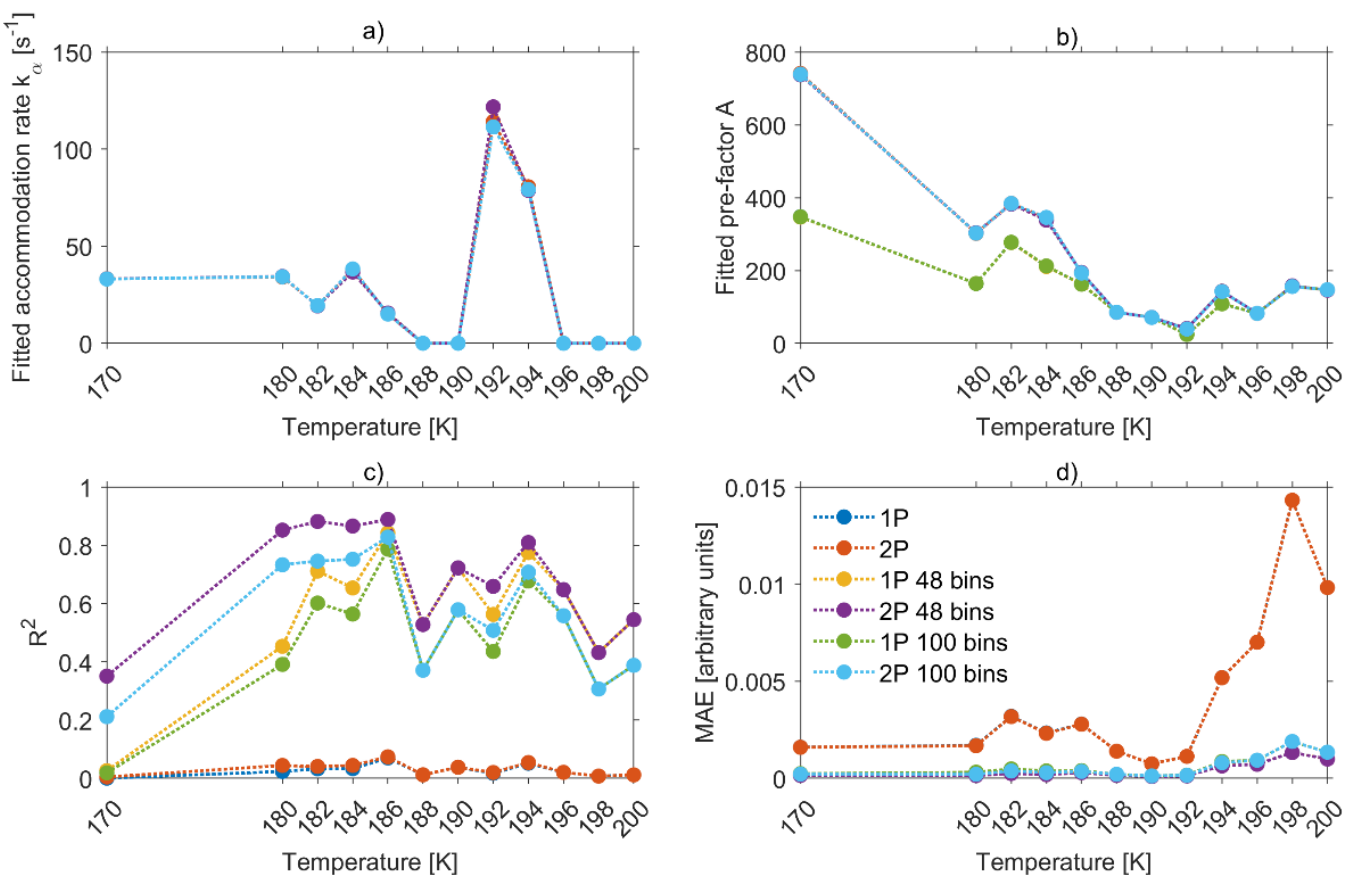

Figure S3: Fitted parameters and associated statistics for modelled $\mathrm{D}_{2} \mathrm{O}$ detection intensities (Figure S2) for the molecular beam data recorded by (Kong et al. 2014). a) fitted accommodation rate of surface layer $\mathrm{D}_{2} \mathrm{O}$ into the bulk crystal of the ice slab using the $2 \mathrm{P}$ flux model. b) fitted pre-factor for $1 \mathrm{P}$ and $2 \mathrm{P}$ flux models. c) $\mathrm{R}^{2}$ statistic. d) Mean absolute error. 


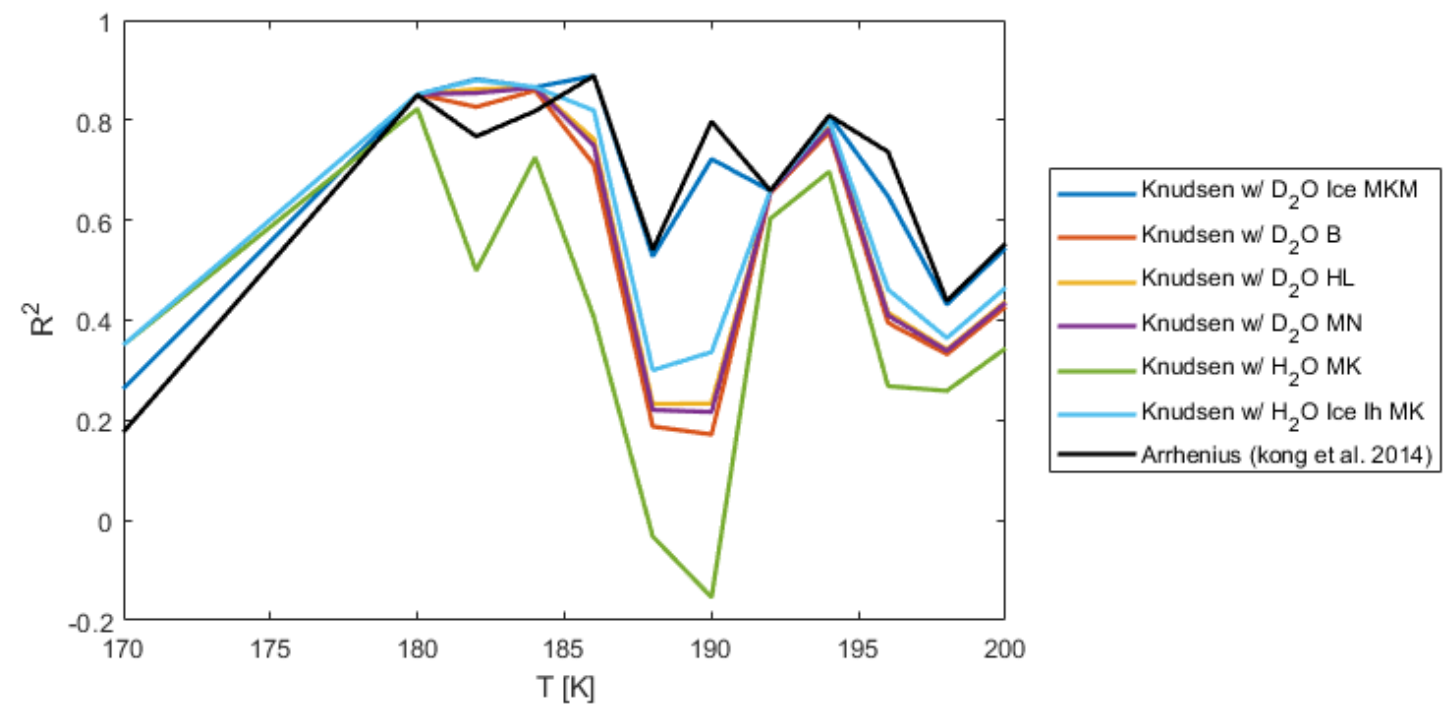

Figure S4: $\mathrm{R}^{2}$-values obtained for fits to the binned experimental EMB data. The Arrheniustype function is the original fit to the data by Kong et al. The abbreviations in the legend are MKM: Matsuo et al., Science $1964^{1}{ }^{\text {; }}$ B: Bhabhe et al, J. Phys. Chem. A, 2013²; HL: Harvey and Lemmon, J. Phys. Chem. Ref. Data, 20023; MN: Matsunaga, Nagashima, Int. J. Thermophys. 19874; MK: Murphy and Koop, Q. J. R. Meteorol.Soc. $2005^{5}$.

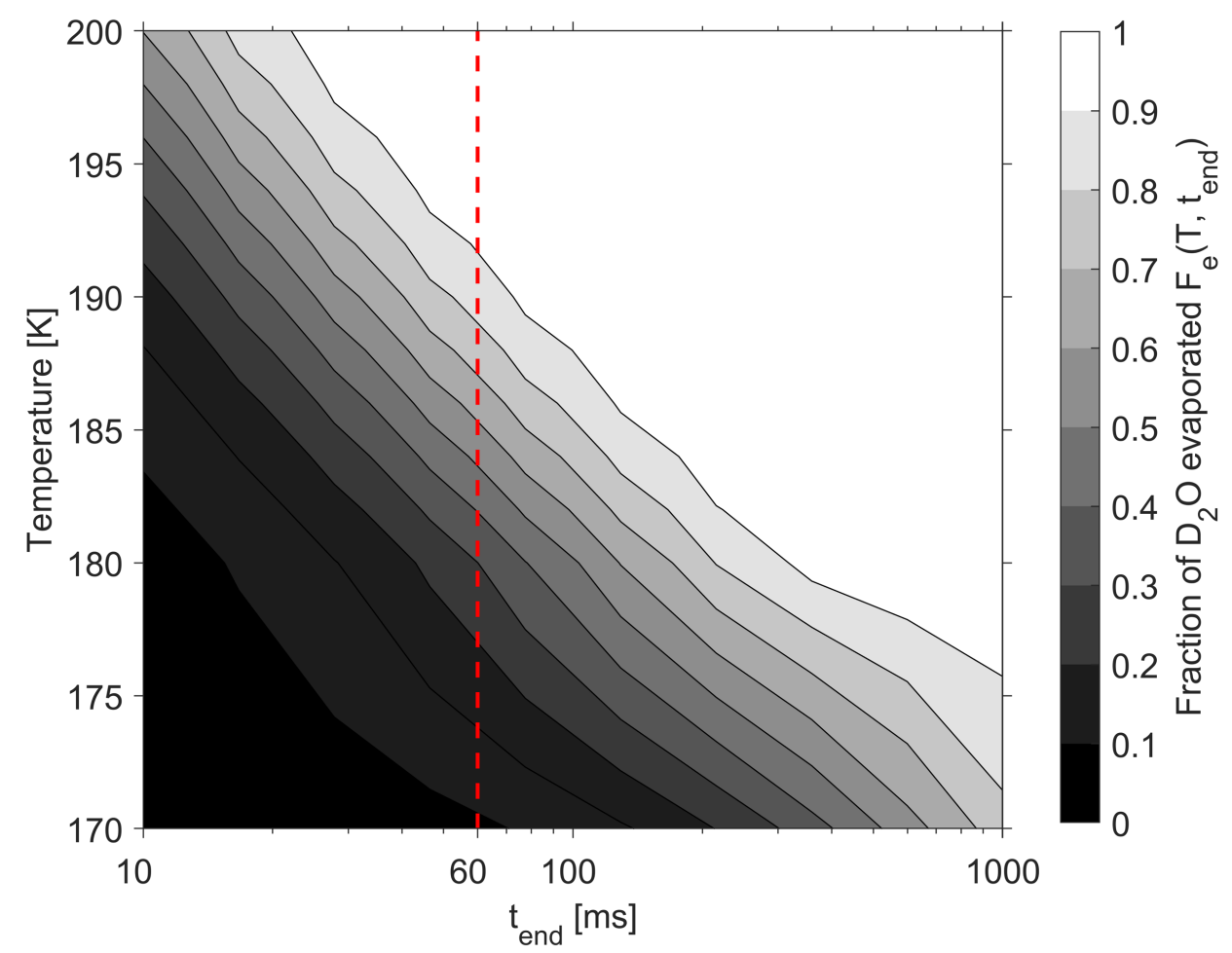

Figure S5: Same as fig. 7 in paper but with Arrhenius-type desorption rate. 
2. Characteristics of energy and order parameter distributions

This paragraph contains additional information about the statistics on short range interaction energy and tetrahedrality parameter distributions of slab molecules (excluding the condensing molecule).

The $10^{\text {th }}, 25^{\text {th }}, 50^{\text {th }}, 75^{\text {th }}$, and $90^{\text {th }}$ percentiles of the probability density of order parameter and short-range interaction energy values for the first layer $(-3 \AA<d<=0 \AA)$ and the third layer $(-9 \AA<d<=-6 \AA)$ were calculated for all temperatures. Tables S1 and S2 contain the $10^{\text {th }}, 25^{\text {th }}$, and median values for the first and third layer, respectively.

Table S1: Definition of different "surface-accommodated" (AC) sub-spaces in E-q-d space. The values are presented for the top-most layer $(-3 \AA<d<=0 \AA)$. The basis for the sub-space definitions is a subdivision into $3 \AA$ layers (about one molecular diameter) parallel to the instantaneous interface, and the numbers in the table are the 10-percentile, the 25-percentile, and the median of energy and order parameter distributions, respectively.

\begin{tabular}{c|c|c|c|c|cc}
\hline & \multicolumn{2}{|c|}{$\begin{array}{c}\text { Surface AC sub-space 1 } \\
(10 \%-i l e)\end{array}$} & \multicolumn{2}{c|}{$\begin{array}{c}\text { Surface AC sub-space 2 } \\
(25 \%-i l e)\end{array}$} & \multicolumn{2}{c}{$\begin{array}{c}\text { Surface AC sub-space } \\
\text { (median) }\end{array}$} \\
\hline$T[\mathrm{~K}]$ & $q$ & $E[\mathrm{~kJ} / \mathrm{mol}]$ & $q$ & $E[\mathrm{~kJ} / \mathrm{mol}]$ & $q$ & $E[\mathrm{~kJ} / \mathrm{mol}]$ \\
\hline 100 & 0.490 & -82.3 & 0.690 & -94.7 & 0.813 & -108.3 \\
170 & 0.617 & -83.3 & 0.727 & -99.3 & 0.827 & -106.7 \\
200 & 0.677 & -79.7 & 0.773 & -93.7 & 0.863 & -104.0 \\
230 & 0.560 & -75.7 & 0.717 & -90.7 & 0.827 & -102.3 \\
250 & 0.473 & -73.0 & 0.677 & -89.0 & 0.803 & -99.7 \\
\hline
\end{tabular}

Table S2: Definition of different "bulk-accommodated" (AC) sub-spaces in $E$ - $q$ - $d$ space for the third slab layer $(-9 \AA<d<=-6 \AA)$. The basis for the sub-space definitions is a subdivision into 3 $\AA$ layers (about one molecular diameter) parallel to the instantaneous interface, and the numbers in the table are the 10-percentile, the 25-percentile, and the median of energy and order parameter distributions, respectively.

\begin{tabular}{c|c|c|c|c|c|c}
\hline & \multicolumn{2}{|c|}{$\begin{array}{c}\text { Bulk AC sub-space 1 } \\
(10 \%-i l e)\end{array}$} & \multicolumn{2}{c|}{$\begin{array}{c}\text { Bulk AC sub-space 2 } \\
\text { (25\%-ile) }\end{array}$} & \multicolumn{2}{c}{$\begin{array}{c}\text { Bulk AC sub-space } \\
\text { (median) }\end{array}$} \\
\hline$T[\mathrm{~K}]$ & $q$ & $E[\mathrm{~kJ} / \mathrm{mol}]$ & $q$ & $E[\mathrm{~kJ} / \mathrm{mol}]$ & $q$ & $E[\mathrm{~kJ} / \mathrm{mol}]$ \\
\hline 100 & 0.937 & -111.0 & 0.953 & -113.7 & 0.970 & -116.0 \\
170 & 0.903 & -103.7 & 0.933 & -108.0 & 0.957 & -111.7 \\
200 & 0.900 & -102.0 & 0.930 & -106.7 & 0.957 & -110.3 \\
230 & 0.887 & -98.7 & 0.920 & -104.0 & 0.950 & -108.7 \\
250 & 0.873 & -97.3 & 0.913 & -102.7 & 0.943 & -107.3 \\
\hline
\end{tabular}


Interestingly, the $10^{\text {th }}$ and $25^{\text {th }}$ percentile values show a non-monotonous temperaturedependence for both $q$ and $E$ at the surface layer, while in the bulk the order parameter decreases and the interaction energy increases monotonously with increasing temperature.

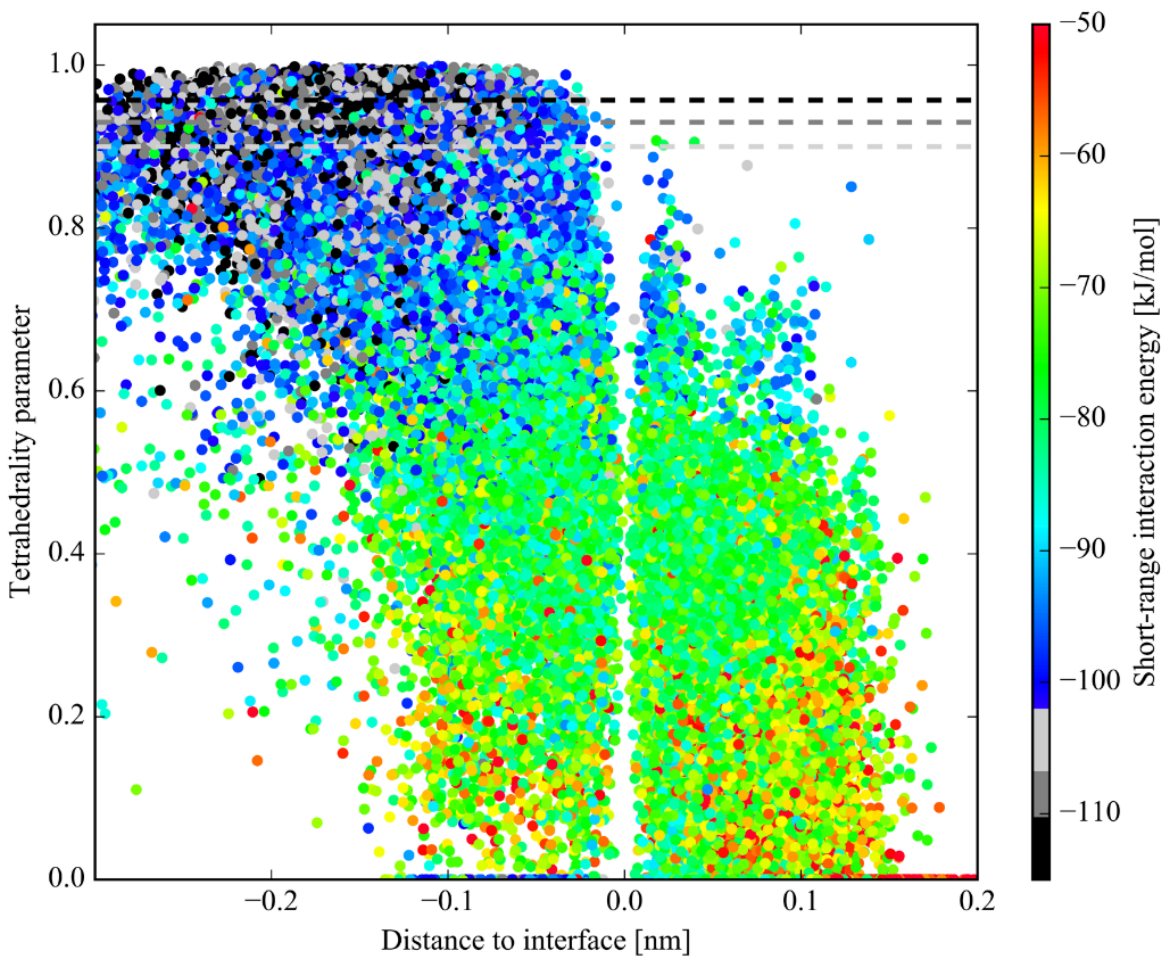

Figure S6: Tetrahedrality parameter $q$ of condensing molecules as a function of distance from the interface for $T=200 \mathrm{~K}$. Positive distances indicate the condensing molecule is located on the gas phase side of the instantaneous interface. Each dot in the scatterplot represents the position of one of 100 condensing molecules in one out of 1000 time frames along the trajectory. The interaction energy $E$ is encoded in the dot's colour. The lines in the plot and the grey scale tones on the colour scale indicate the cut-off criteria for the tetrahedrality parameter and interaction energy, respectively for sub-spaces AC1: light gray, AC2: gray, AC: black (see Table S2 for the definition of Bulk AC sub-spaces). For a molecule to be considered inside a sub-space, the criteria for both tetrahedrality parameter and interaction energy must be fulfilled.

\section{Time correlation functions of condensing molecules}

Time correlation functions of interaction energy and tetrahedrality parameter of the condensing molecules were calculated as $\left\langle\left|\frac{E_{\text {cond }}(t)}{\langle E\rangle}-1\right|\right\rangle_{\text {runs }}$, where $\langle E\rangle$ denotes the expectation value of the interaction energy of molecules in the topmost $3 \AA$ layer, $E_{\text {cond }}(t)$ denotes the interaction energy of the condensing molecule, and the average $\langle\bullet\rangle_{\text {runs }}$ is taken over the ensemble of 100 simulations (analogously for $q$ ). Estimates of the characteristic time 
scales of time correlation functions show two regimes: A short- and a long-time decay. The short- and long-time behaviours, defined as up to 100 ps and from 500 ps onwards, were fit with an exponential function of the form $C(t)=a e^{-b t}$, respectively, where the characteristic time scale is defined as $1 / b$.
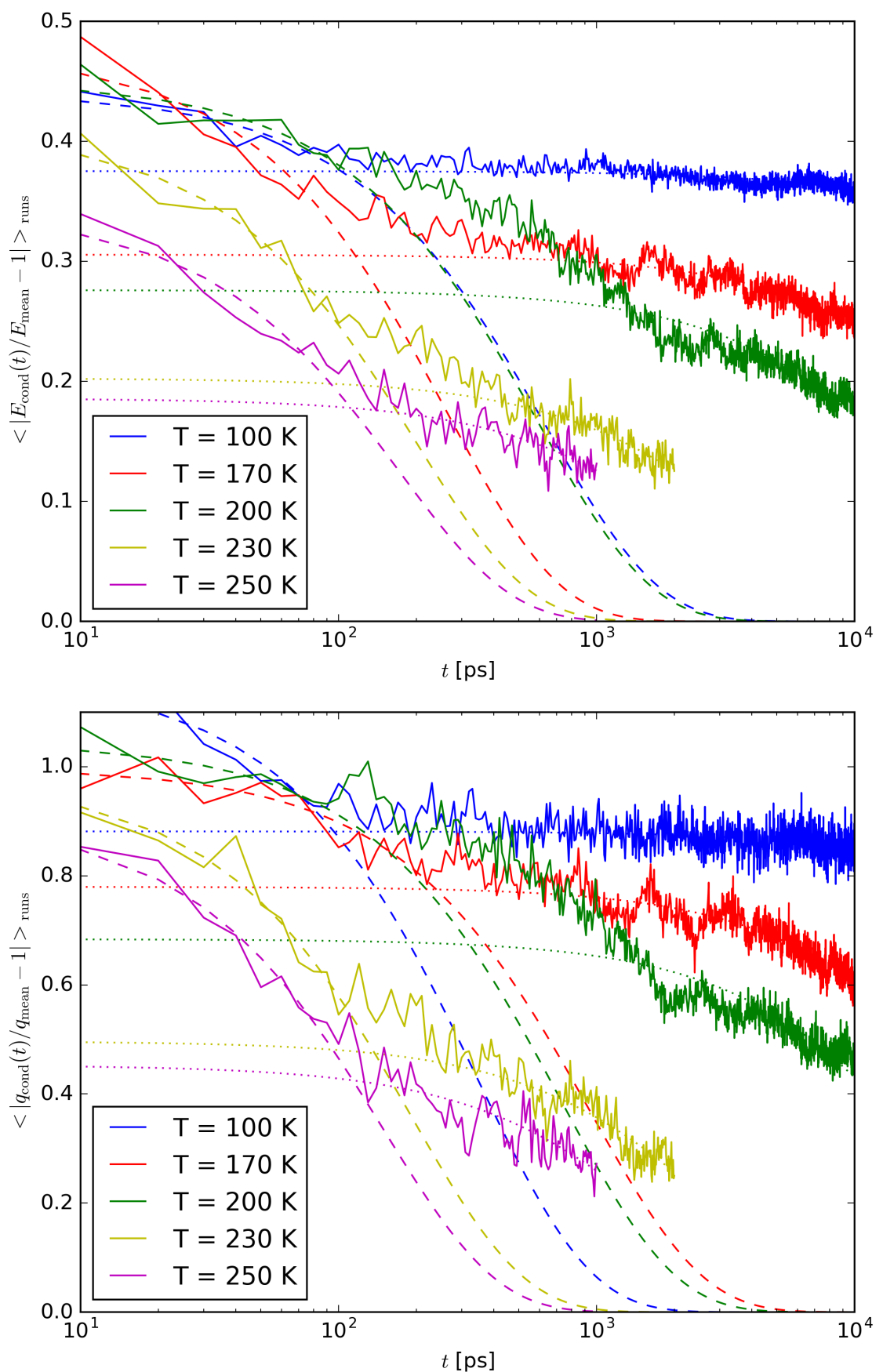

Figure S7: Time correlation functions of interaction energy and tetrahedrality parameter of condensing molecules. The fits are exponentials of the form $C(t)=a e^{-b t}$ with fitting parameters $a, b$. The value of $1 / b$ is taken as the characteristic time of the decay. Correlation 
functions were fit to the short- (dashed lines) and the long-time (dotted lines) behaviour, i.e. up to 100 ps and from 500 ps onwards, respectively.

Table S3: Characteristic time scales of the time correlation functions of condensing molecules obtained from exponential fits to the short- and long-time behaviours of $E$ - and $q$ time correlation functions. Uncertainty ranges represent standard deviations obtained from the covariance matrices of the least-squares fits.

\begin{tabular}{|c|c|c|c|c|}
\hline $\mathrm{T}[\mathrm{K}]$ & $\begin{array}{c}1 / b_{q}[p s] \\
\text { Fit } 1-100 p s\end{array}$ & $\begin{array}{l}1 / \mathrm{b}_{\mathrm{q}}[\mathrm{ns}] \\
\text { Fit from } 0.5 \mathrm{~ns}\end{array}$ & $\begin{array}{c}1 / b_{E}[p s] \\
\text { Fit } 1-100 \text { ps }\end{array}$ & $\begin{array}{c}1 / b_{E}[n s] \\
\text { Fit from } 0.5 \text { ns }\end{array}$ \\
\hline 100 & $350_{-40}^{+40}$ & $270_{-25}^{+27}$ & $640_{-100}^{+140}$ & $231_{-9}^{+11}$ \\
\hline 170 & $950_{-250}^{+520}$ & $39_{-0.7}^{+0.6}$ & $260_{-40}^{+60}$ & $\sim 47$ \\
\hline 200 & $740_{-140}^{+210}$ & $21.6_{-0.5}^{+0.4}$ & $600_{-120}^{+190}$ & $20.7_{-0.3}^{+0.4}$ \\
\hline 230 & $190_{-20}^{+30}$ & $3.0_{-0.1}^{+0.2}$ & $200_{-20}^{+30}$ & $4.2_{-0.3}^{+0.2}$ \\
\hline 250 & $150_{-10}^{+10}$ & $1.8_{-0.3}^{+0.4}$ & $170_{-20}^{+30}$ & $2.6_{-0.5}^{+0.6}$ \\
\hline
\end{tabular}

Table S3 shows the characteristic time scales for the decay of the time correlation functions. We note that the short-time decay exhibits a non-monotonous trend with temperature while the long-time decay takes increasingly longer time with decreasing temperature. Characteristic long-time decay scales of $E$ and $q$ are found to agree well.

4. Frequency analysis of exchange processes between accommodated and nonaccommodated states

Using the criteria for accommodation defined in the paper, we find that even bulk ice molecules fluctuate between accommodated and non-accommodated states. The analysis of the exchange frequency shows that bulk molecules exhibit a higher frequency than molecules in the surface layer, see Tab. S4 and Fig. S8. We interpret this finding as a result of stronger bonds at crystal sites than at the surface, in analogy to the frequency of a harmonic oscillator depending on the force constant. This picture also explains the trend with temperature since indeed the mean square displacement of molecules about crystal sites due to thermal motion is directly proportional to temperature, assuming a harmonic potential. Due to time binning during analysis, the maximum frequency detectable here is $\mathrm{f}_{\mathrm{ex}, \max }=100 \mathrm{~ns}^{-1}$. 
Table S4: Averages of exchange frequencies between accommodated and nonaccommodated states for all, bulk molecules and surface molecules

\begin{tabular}{|c|c|c|c|}
\hline $\mathbf{T}[K]$ & $\begin{array}{c}\text { All molecules } \\
\mathbf{f}_{\mathbf{e x}}\left[\mathbf{n s}^{-1}\right]\end{array}$ & $\begin{array}{c}\text { Bulk molecules } \\
-\mathbf{0 . 8} \mathbf{n m}<\mathbf{d}<-\mathbf{0 . 5} \mathbf{n m} \\
\mathbf{f}_{\mathrm{ex}}\left[\mathbf{n s}^{-1}\right]\end{array}$ & $\begin{array}{c}\text { Surface molecules } \\
-\mathbf{0 . 3} \mathbf{n m}<\mathbf{d}<\mathbf{0 . 0} \mathbf{n m} \\
\mathbf{f}_{\mathbf{e x}}\left[\mathbf{n s}^{-1}\right]\end{array}$ \\
\hline $\mathbf{1 0 0}$ & 6.0 & 8.5 & 0.2 \\
\hline $\mathbf{1 7 0}$ & 9.8 & 11.7 & 0.8 \\
\hline $\mathbf{2 0 0}$ & 10.6 & 12.3 & 1.6 \\
\hline $\mathbf{2 3 0}$ & 10.5 & 13.2 & 2.1 \\
\hline $\mathbf{2 5 0}$ & 9.1 & 9.6 & 2.4 \\
\hline
\end{tabular}

Table S5: Rough estimates of the accommodation rate obtained from the first passage time (FPT) analysis, see Fig. 6 of the paper. These numbers are obtained from the slope of linear fit to the cumulative FPT distributions.

\begin{tabular}{|c|c|c|}
\hline $\mathbf{T}[\mathrm{K}]$ & $\begin{array}{c}\text { Accommodation rate, } \\
\text { first layer criteria } \\
\boldsymbol{\gamma}_{\boldsymbol{e x}}\left[\mathrm{ns}^{-1} \text { ] }\right.\end{array}$ & $\begin{array}{c}\text { Accommodation rate, } \\
\text { third layer criteria } \\
\boldsymbol{\gamma}_{\boldsymbol{e x}}\left[\mathrm{ns}^{-1}\right]\end{array}$ \\
\hline $\mathbf{1 0 0}$ & 0.1 & -- \\
\hline $\mathbf{1 7 0}$ & 7.5 & 0.5 \\
\hline $\mathbf{2 0 0}$ & 8.1 & 5.3 \\
\hline $\mathbf{2 3 0}$ & 43 & 26 \\
\hline $\mathbf{2 5 0}$ & 100 & 36 \\
\hline
\end{tabular}

The approximation of harmonic potentials becomes worse close to the surface and at higher temperatures when anharmonic effects have to be expected. Comparing rough estimates of frequencies from the first passage time analysis with the frequency of exchange processes we conclude that they are of the same order of magnitude with the general trend of increasing frequencies with increasing temperature. At the highest temperature of $\mathrm{T}=250 \mathrm{~K}$, exchange frequencies of bulk molecules decrease compare to $T=230 \mathrm{~K}$. This is speculated to be due to surface effects gradually reaching down to what in this analysis was defined as "bulk" with increasing temperature. 


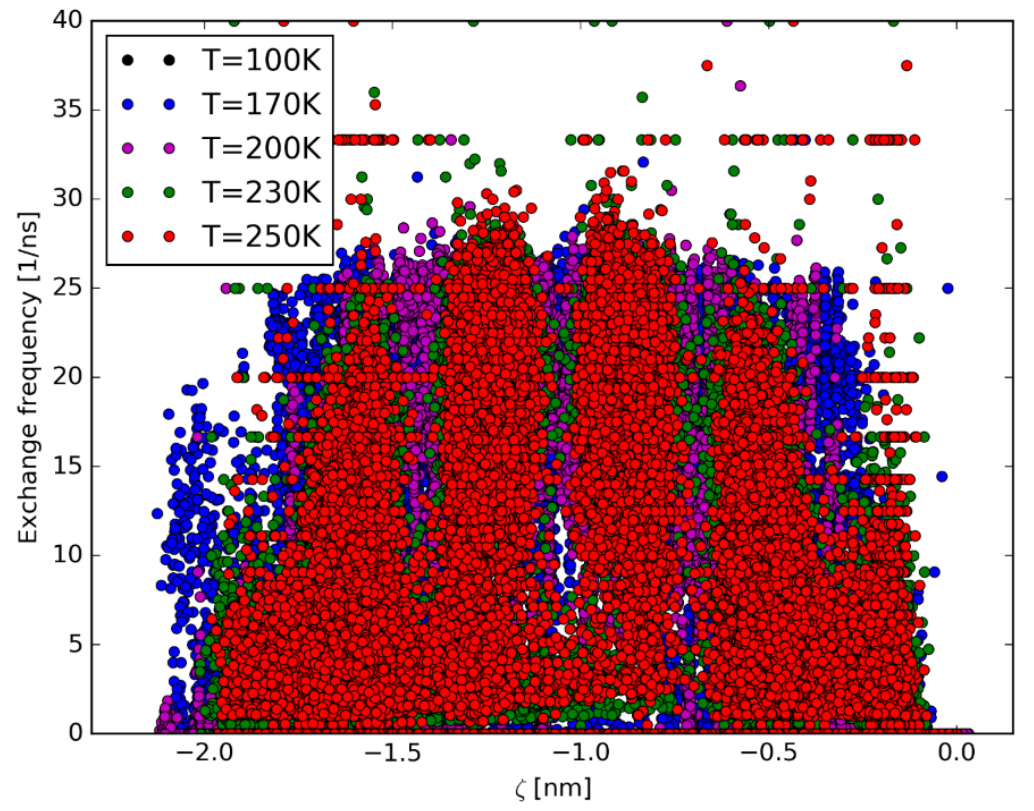

Figure S8: Exchange frequency between $A C$ and NAC states depending on the distance to the instantaneous interface.

The histogram of the exchange frequencies at different temperatures, Fig. S9, shows a bimodal shape with a minimum at intermediate values around $12 \mathrm{~ns}^{-1}$ and a relatively sharp cut-off around $27 \mathrm{~ns}^{-1}$ which we haven't been able to explain conclusively yet. From the histogram it also becomes quite clear that average values are of limited use when analysing the exchange frequency data.

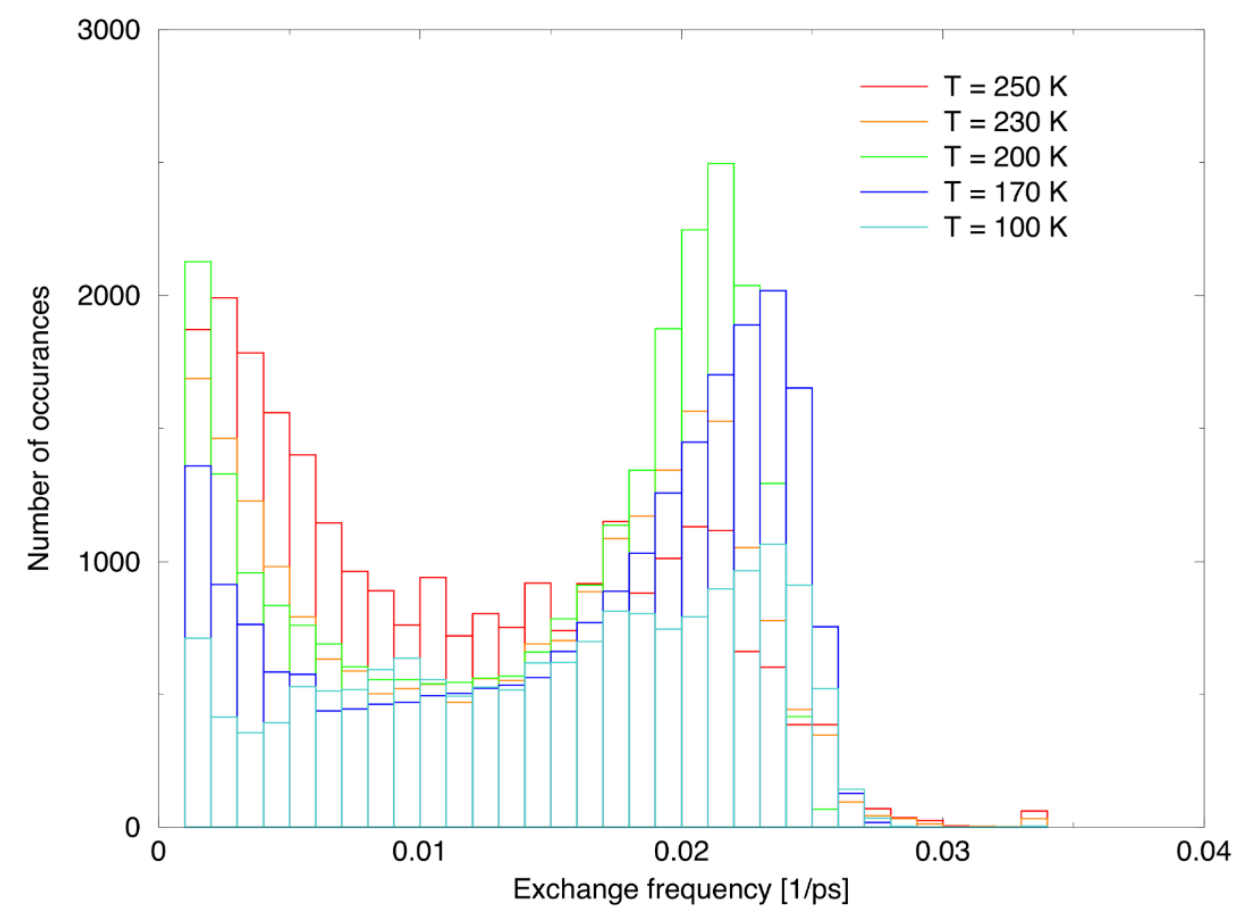

Figure S9: Histogram of exchange frequencies between accommodated and nonaccommodated states (excluding interval $0.0-0.001 \mathrm{ps}^{-1}$ ). 
(1) Matsuo, S.; Kuniyoshi, H.; Miyake, Y. Vapor Pressure of Ice Containing D2O. Science 1964, 145 (3639), 1454-1455. https://doi.org/10.1126/science.145.3639.1454.

(2) Bhabhe, A.; Pathak, H.; Wyslouzil, B. E. Freezing of Heavy Water (D2O) Nanodroplets. J. Phys. Chem. A 2013, 117 (26), 5472-5482. https://doi.org/10.1021/jp400070v.

(3) Harvey, A. H.; Lemmon, E. W. Correlation for the Vapor Pressure of Heavy Water From the Triple Point to the Critical Point. J. Phys. Chem. Ref. Data 2002, 31 (1), 173-181. https://doi.org/10.1063/1.1430231.

(4) Matsunaga, N.; Nagashima, A. Saturation Vapor Pressure and Critical Constants of $\mathrm{H} 2$ O, D 2 O, T 2 O, and Their Isotopic Mixtures. Int. J. Thermophys. 1987, 8 (6), 681-694.

(5) Murphy, D. M.; Koop, T. Review of the Vapour Pressures of Ice and Supercooled Water for Atmospheric Applications. Q. J. R. Meteorol. Soc. 2005, 131 (608), 1539-1565. https://doi.org/10.1256/qj.04.94. 